\title{
Compression of matter in the center of accreting neutron stars
}

\author{
M. Bejger ${ }^{1}$, J. L. Zdunik ${ }^{1}$, P. Haensel ${ }^{1}$, and M. Fortin ${ }^{1,2}$ \\ 1 N. Copernicus Astronomical Center, Polish Academy of Sciences, Bartycka 18, 00-716 Warszawa, Poland \\ e-mail: [bejger; jlz; haensel]@acamk.edu.pl \\ 2 LUTh, UMR 8102 du CNRS, Observatoire de Paris, 92195 Meudon Cedex, France \\ e-mail: morgane.fortin@obspm.fr
}

Received 5 September 2011 / Accepted 26 October 2011

\begin{abstract}
Aims. To estimate the feasibility of dense-matter phase transition, we studied the evolution of the central density as well as the baryon chemical potential of accreting neutron stars. We compared the thin-disk accretion with and without the magnetic field torque with the spin-down scenario for a selection of recent equations of state.

Methods. We compared the prevalent (in the recycled-pulsar context) Keplerian thin-disk model, in which the matter is accreted from the marginally-stable circular orbit, with the recent magnetic-torque model that takes into account the influence of stellar magnetic field on the effective inner boundary of the disk. Calculations were performed using a multi-domain spectral methods code in the framework of General Relativity. We considered three equations of state consistent with the recently measured mass of PSR J1614$2230,1.97 \pm 0.04 M_{\odot}$ (one of them softened by the appearance of hyperons).

Results. If there is no magnetic torque and efficient angular momentum transfer from the disk to the star, substantial central compression is limited to the region of initial stellar masses close to the maximum mass. Outside the maximum mass vicinity, accretion-induced central compression is significant only if the angular momentum transfer is inefficient. Accounting for the magnetic field effectively decreases the efficiency of angular momentum transfer and implies a significant central compression.

Conclusions. An efficient angular momentum transfer from a thin disk onto a non-magnetized neutron star does not provide a good mechanism for the central compression and possible phase transition. Substantial central compression is possible for a broad range of masses of slowly-rotating initial configurations for magnetized neutron stars. Accretion-induced central compression is particularly strong for stiff equation of state with a high-density softening.
\end{abstract}

Key words. stars: neutron - accretion, accretion disks - magnetic fields - pulsars: general - dense matter

\section{Introduction}

Neutron stars (NSs) are detected in binary systems by X- and $\gamma$-ray observatories because of the immense amounts of radiation released in the process of accretion of matter falling from a companion star onto the NS surface. Because the stellar mass increases during accretion, it is usually assumed that the density in the center of the star increases as well, and that a sufficient increase of mass may result in a phase transition (the details of which are not yet fully known). It is expected that a phase transition in the NS core might cause observable astrophysical phenomena, such as star-quakes, spin clustering, gravitational wave emission and therefore serve, it is hoped, as a testbed for the quantum chromodynamics (QCD) phase diagram (Cheng \& Dai 1998; Glendenning \& Weber 2001; Blaschke et al. 2001; Zdunik et al. 2005; Blaschke et al. 2008; Xu \& Liang 2009 and references therein).

We aim to show with three recent equations of state (EOSs) of NS cores, that the central baryon number density, $n_{\mathrm{c}}$, and the baryon chemical potential, $\mu_{\mathrm{c}}$, may change in a non-trivial way while the star gains in mass and simultaneously increases its rotation rate during accretion. In particular, we will study whether, and if so, to what extent, the evolution of $n_{\mathrm{c}}$ and $\mu_{\mathrm{c}}$ depends on the EOS of dense matter. Our considered EOSs are consistent with the recently-measured mass of PSR J16142230, $1.97 \pm 0.04 M_{\odot}$ (Demorest et al. 2010). Complementary questions that may be asked are (a) how does the actual efficiency of the angular-momentum transfer and the stellar magnetic field coupling to the disk affect the final rapidly-rotating NS configuration; and (b) how does the accretion-induced evolution depend on the mass of the initially slowly rotating configuration. Thus far, the formation of millisecond pulsars was most often modeled following the classical paper of Cook et al. (1994) who assumed the stellar magnetic field is sufficiently weak that it does not influence the accretion flow significantly; consequently, one assumed $B=0$ and the accretion from the marginally-stable circular orbit (of radius $r_{\mathrm{ms}}$ ). Notable exceptions were Possenti et al. (1999), Burderi et al. (1999), Blaschke et al. (2001), Colpi et al. (2001), and Blaschke et al. (2008), who used simple models of magnetic torque acting in the NS-accretion-disk system (Ghosh \& Lamb 1979, see also Ghosh \& Lamb 1991).

Here we compare the results obtained using the $B=0$ approach with those employing the recent effective magnetictorque model of Kluźniak \& Rappaport (2007), used recently by Bejger et al. (2011) to study the formation of a millisecond pulsar PSR 1903+0327. The details of magnetic-field decay during the accretion-driven evolution phase of an NS' life are still far from being understood in spite of theoretical and observational efforts in recent years (see Sect. 4 of Bejger et al. 2011 for a brief summary). Hence, we employ the simplest possible description of the magnetic-field decay that was shown to be consistent with 
at least a subclass of observed accreting NSs (Shibazaki et al. 1989) - this approach allows us to study the qualitative features of the influence of disk accretion on the behavior of the NS central parameters.

The text is organized in the following way. Section 2 gives a short description of the methods used to obtain the accreting NS tracks. The results are presented in Sect. 3. Section 4 contains our summary and conclusions.

\section{Methods and models}

We simulated the spin evolution of NSs caused by the thin-disk accretion without a magnetic field and compared it with the results for the magnetic-torque model of Kluźniak \& Rappaport (2007). Because the original Newtonian magnetic-torque model was insufficient in extreme cases of rapidly-rotating, massive and compact NSs, we additionally employed the modification by Bejger et al. (2011), which takes into account the relativistic effects caused by the existence of the marginally-stable circular orbit (recalled briefly in the Appendix). We considered three recent EOSs - APR EOS by Akmal et al. (1998), DH EOS by Douchin \& Haensel (2001) and BM EOS from a set of non-linear relativistic mean field models of Bednarek \& Mańka (2009) ${ }^{1}$. The first two EOSs assume nucleon NS cores, while the BM EOS has a characteristic high-density softening associated with appearance of hyperons. Constructing an EOS yielding $M_{\max }>1.97 M_{\odot}$ in spite of the hyperon softening is not an easy task, therefore it deserves some additional explanations. The crucial feature of our BM EOS is a repulsive contribution to the pressure coming from the quartic terms in the vector meson fields in the Lagrangian (Bednarek \& Mańka 2009). We emphasize, that the BM model reproduces semi-empirical nuclear-matter data as well as the semi-empirical estimates of the potential wells of hyperons in the nuclear matter, coming from hypernuclear data, involving hypernuclei and $\Sigma^{-}$-atoms, as well as the strength of the $\Lambda-\Lambda$ attraction in the $\Lambda \Lambda$-hypernuclei (Bednarek \& Mańka 2009). Neutron-star configurations were obtained with the numerical library LORENE ${ }^{2}$, using the implementation of the Bonazzola et al. (1993) formalism for axisymmetric and rigidly-rotating stars (rotstar code).

\subsection{Magnetic torque neglected}

Assuming that the magnetic field is not disturbing the Keplerian thin accretion disk structure, one usually considers the spin-up scenario in which the angular momentum is transferred from the disk to the NS via accretion from the marginally-stable circular orbit (Cook et al. 1994; Zdunik et al. 2002). In this process, an infall of a particle of baryon mass $\mathrm{d} M_{\mathrm{b}}$ and specific orbital angular momentum $l_{\mathrm{ms}}$ leads to a new quasi-stationary stellar configuration of baryon mass $M_{\mathrm{b}}+\mathrm{d} M_{\mathrm{b}}$ and angular momentum $J+\mathrm{d} J$, according to

$$
\frac{\mathrm{d} J}{\mathrm{~d} M_{\mathrm{b}}}=x_{\mathrm{l}} l_{\mathrm{ms}}
$$

where the parameter $x_{1}(\leq 1)$ quantifies our lack of knowledge of the fraction of angular momentum that is transferred to the

\footnotetext{
1 We use a specific model corresponding to the parameter $\Lambda_{\mathrm{V}}=0.016$. It fits semi-empirical hypernuclear and nuclear data and yields for an NS rotating at $317 \mathrm{~Hz}$ (spin frequency of PSR J1614-2230) $M_{\max }=$ $2.01 M_{\odot}$.

2 http://www . lorene.obspm. fr
}

star by an infalling particle; recent numerical simulations suggest that the value of $x_{1}$ is close to unity (see, e.g., Beckwith et al. 2008; Shafee et al. 2008).

\subsection{Magnetic torque included}

If the magnetic field does affect the accretion flow, the $B=0$ model ceases to be correct. To calculate the increase of the total stellar angular momentum $J$ in this case, one must modify the Eq. (1) to incorporate the fact that the disk now terminates at some $r_{0}>r_{\mathrm{ms}}$, depending on the value of magnetic field. We use the prescription of Kluźniak \& Rappaport (2007), which gives the following evolution equation:

$$
\frac{\mathrm{d} J}{\mathrm{~d} M_{\mathrm{b}}}=l\left(r_{0}\right)-\frac{\mu^{2}}{9 r_{0}^{3} \dot{M}_{\mathrm{b}}}\left[3-2\left(\frac{r_{\text {cor }}}{r_{0}}\right)^{3 / 2}\right],
$$

where $\mu=B R^{3}$ is the dipole magnetic moment of an NS (assumed to be parallel to $J$ ), $r_{\text {cor }}$ is the corotation radius and $\dot{M}_{\mathrm{b}}$ denotes the mean accretion rate; for a detailed description of the parameters and modifications in obtaining $r_{0}$, related to the relativistic marginally-stable circular orbit, see the appendix and Bejger et al. (2011).

A complete evolution model of a magnetized NS accreting from a disk needs a formula for the accretion-induced surface magnetic field decay. Because the theoretical state-of-the-art of this subject is quite complicated and far from being complete (see Sect. 4 of Bejger et al. 2011 for a brief summary), we used the simplest, but observationally-motivated decay law proposed by Shibazaki et al. (1989; see also Taam \& van den Heuvel 1986; van den Heuvel \& Bitzaraki 1995) and assumed that $B$ decreases as a function of accreted mass $\Delta M$ only: $B=B_{\mathrm{i}} /\left(1+\Delta M / m_{B}\right)$, where $m_{B}=10^{-4} M_{\odot}$. We also tested other available forms of decay law (exponential-like formula used recently by Osłowski et al. 2011 in the context of population-synthesis studies, and the quadratic modification of Shibazaki et al. law) and conclude that this choice has no qualitative influence on the results.

\section{Results}

To estimate the feasibility of dense-matter phase transitions, we examined the compression of matter in the cores of accreting NSs by measuring the increase of the central baryon number density $n_{\mathrm{c}}$ as well as the central baryon chemical potential $\mu_{\mathrm{c}}=\left(p_{\mathrm{c}}+e_{\mathrm{c}}\right) / n_{\mathrm{c}}\left(p_{\mathrm{c}}\right.$ and $e_{\mathrm{c}}$ are the pressure and mass-energy density, respectively). Furthermore, we compared the results for spin-up-induced central compression with the isolated NS spindown results.

\subsection{Magnetic torque neglected}

For $B=0$ the features of accretion-driven evolution of $n_{\mathrm{c}}$ and $\mu_{\mathrm{c}}$ are qualitatively similar for all investigated EOSs. We will thus illustrate these features using the DH EOS and begin with the comparison of spin-up (with the most efficient angular momentum transfer $x_{1}=1$ ) and spin-down-induced increase of $n_{\mathrm{c}}$ pictured in Fig. 1 in the spin-frequency-central density plot; the configurations are allowed to span the whole frequency range from the initially non-rotating stars up to the mass-shedding limit (or the axi-symmetric perturbation instability limit). Evidently, the spin-down allows for a higher $n_{\mathrm{c}}$ increase than the $x_{1}=1$ accretion spin-up. To investigate this feature, Fig. 2 shows the behavior of the gravitational mass $M$ as a 


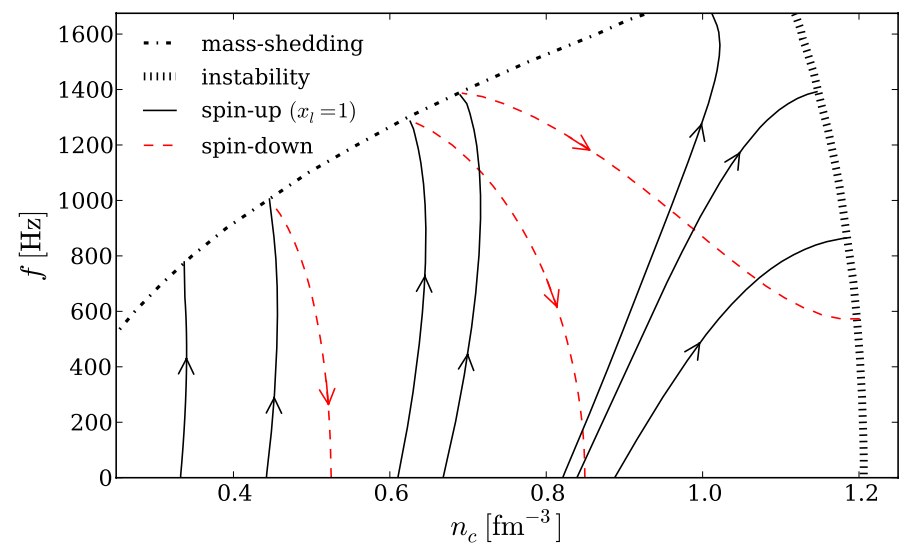

Fig. 1. Evolution of the central baryon number density $n_{\mathrm{c}}$ in the diskaccretion spin-up as well as the spin-down scenario, shown for the spin frequency $f$-central baryon density $n_{\mathrm{c}}$ plane (DH EOS, $B=0$ ).

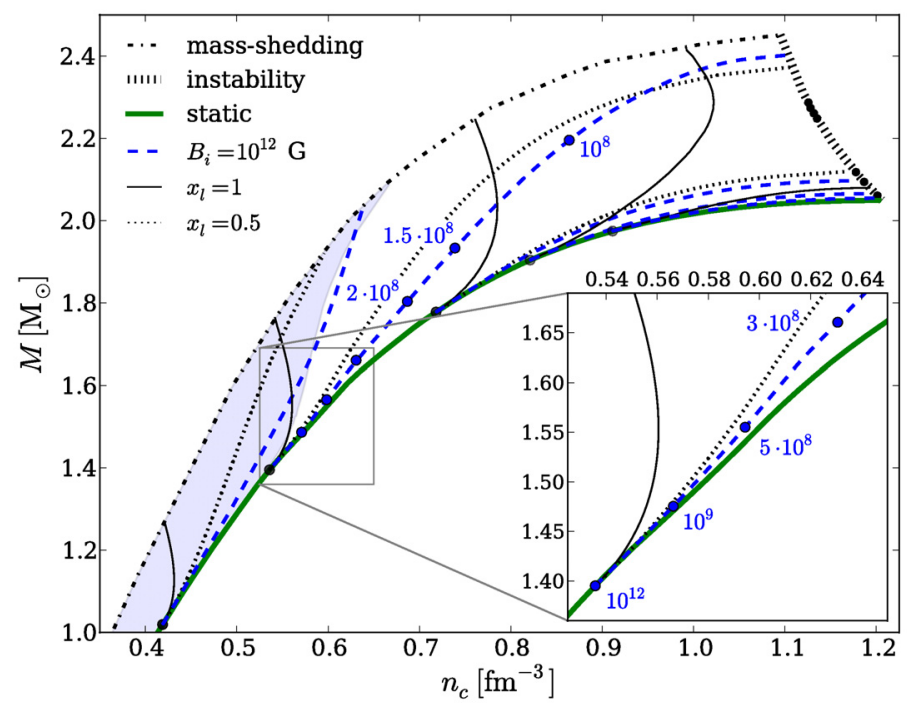

Fig. 2. Accretion tracks of the gravitational mass-central baryon number density, $M-n_{\mathrm{c}}$, plane. Magnetic field tracks were obtained for $\dot{M}_{\mathrm{b}}=10^{-9} M_{\odot} / \mathrm{yr}$. The shaded region contains configurations for which the stellar equatorial radius $R_{\mathrm{eq}}$ is larger than $r_{\mathrm{ms}}$ (DH EOS).

function of $n_{\mathrm{c}}$. For an astrophysically-motivated range of NS initial masses, $M_{\mathrm{i}}=1.2-1.6 M_{\odot}$, the $x_{1}=1$ accretion never leads to a substantial central compression; moreover, the maximal central compression is attained for moderate spin periods, followed by a decrease of $n_{\mathrm{c}}$. On the contrary, lowering the efficiency of angular momentum transfer to e.g. $x_{1}=0.5$ allows for a substantial $n_{\mathrm{c}}$ increase. A detailed summary and comparison between various types of tracks is included in Fig. 3, where the initial and final $n_{\mathrm{c}}$ is plotted as a function of the initial stellar mass $M_{\mathrm{i}}$ for both spin-up (upper panel) and spin-down (lower panel). The nearly horizontal line for $x_{1}=0.5$ in the upper panel denotes the instability limit. Based on these data we conclude that to achieve a significant $n_{\mathrm{c}}$ increase in an astrophysically-sound evolutionary process one should consider

- an initial mass of the configuration quite close to the value of the static-star maximum mass in case of $x_{1}=1$,

- or a value of $x_{1}$ significantly lower than 1 (dotted lines in Figs. 2 and 3),

- a more efficient mechanism, such as the spin-down of an isolated NS - constant $M_{\mathrm{b}}$ track leads to a higher increase of
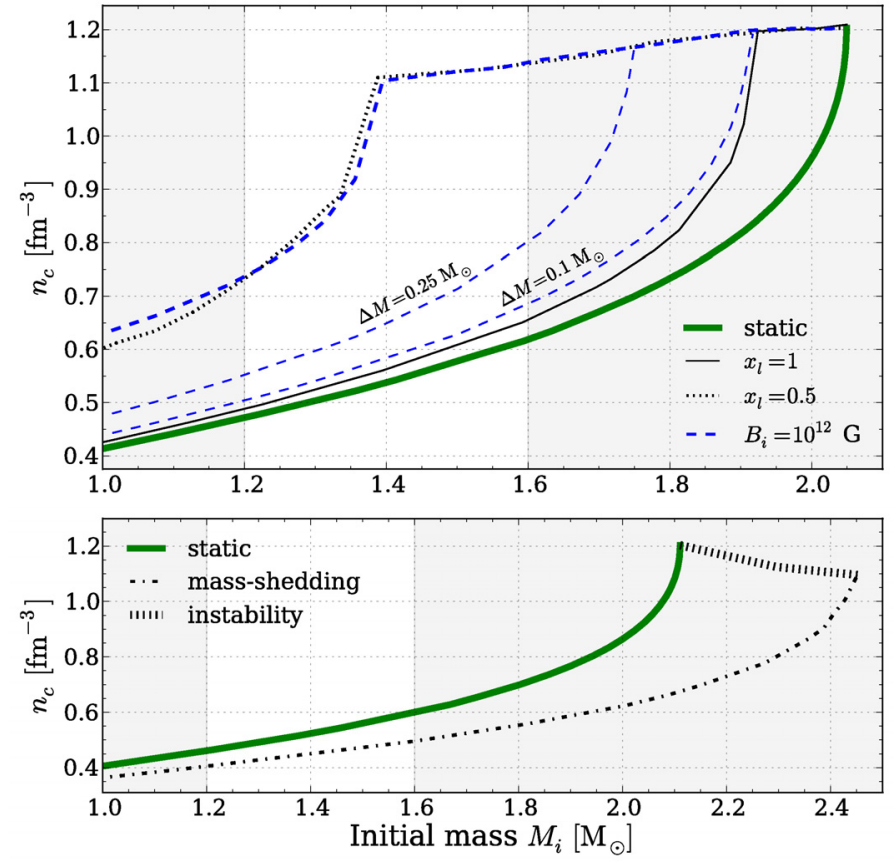

Fig. 3. Maximum central density $n_{\mathrm{c}, \mathrm{f}}$ attainable for a given evolutionary track compared to the initial $n_{\mathrm{c}, \mathrm{i}}$, both plotted as functions of the gravitational mass of the initial non-rotating configuration, $M_{\mathrm{i}}$. Upper panel: spin-up scenario. The reference line $n_{\mathrm{c}, \mathrm{i}}$ is the static sequence. Lower panel: spin-down scenario. The reference $n_{\mathrm{c}, \mathrm{i}}$ line is the mass-shedding limit, to be compared with $n_{\mathrm{c}, \mathrm{f}}$ of static configurations (DH EOS).

$n_{\mathrm{c}}$ than thin-disk accretion, as shown in Fig. 1 and the lower panel of Fig. 3.

Results for the chemical potential $\mu_{\mathrm{c}}$ for the DH EOS are plotted in Fig. 4; results for APR and BM EOSs are presented in Figs. 5 and 6.

\subsection{Magnetic torque included}

An inclusion of the magnetic torque acting in the NS-disk system changes the results significantly, as Fig. 2 shows. As an example, we used the "canonical case" of the initial magnetic field $B_{\mathrm{i}}=10^{12} \mathrm{G}$ and the average accretion rate $\dot{M}_{\mathrm{b}}=10^{-9} M_{\odot} / \mathrm{yr}$ (dashed lines; dots denote the decaying magnetic field for the initial mass $M_{\mathrm{i}}=1.4 M_{\odot}$ ). Overall, the magnetic-torque results resemble those for a reduced efficiency of the angular momentum transfer, $x_{1}=0.5$. Including the magnetic field substantially increases $n_{\mathrm{c}}$ and $\mu_{\mathrm{c}}$ for an astrophysically interesting range of initial masses, $M_{\mathrm{i}}=1.2-1.6 M_{\odot}$ (Figs. 3 and 4 for the DH EOS, and Figs. 5 and 6 for APR and BM EOSs). The nearly horizontal line segments in the upper panels of Figs. 3 and 5 correspond to the axisymmetric instability limit - it is reached by configurations that were not spun up to the mass-shedding limit; lowering the mass accretion rate results in an extended instability limit line toward smaller initial masses. In addition to the maximum attainable compression, the results for a predefined amount of accreted mass $\left(\Delta M=0.1 M_{\odot}, \Delta M=0.25 M_{\odot}\right)$ are plotted for comparison in Fig. 3.

For the DH EOS and $M_{\mathrm{i}}=1.4 M_{\odot}$, accretion of about $0.25 M_{\odot}$ implies $\left(n_{\mathrm{c}, \mathrm{f}}-n_{\mathrm{c}, \mathrm{i}}\right) / n_{\mathrm{c}, \mathrm{i}} \approx 0.2$. This should be compared with very small (of a few percent) compression for $B=0$ and $x_{1}=1$ case. Including the magnetic torque is therefore crucial for accretion-induced compression in the core of an NS with initial mass $\sim 1.4 M_{\odot}$. Note also a fairly strong effect of the EOS 

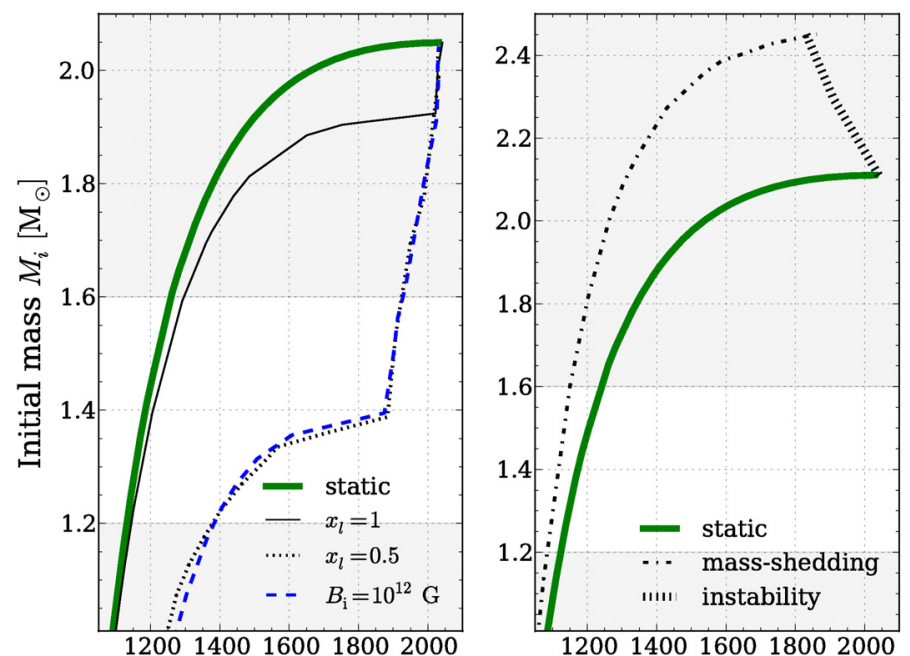
$\mu_{c}[\mathrm{MeV}]$

Fig. 4. Gravitational mass of the initially non-rotating configuration, $M_{\mathrm{i}}$ as a function of the maximum attainable central baryon chemical potential $\mu_{\mathrm{c}, \mathrm{f}}$ for a given evolutionary track compared to the initial $\mu_{\mathrm{c}, \mathrm{i}}$. Lines are denoted analogously to Fig. 3. Left panel: spin-up scenario. Right panel: spin-down scenario (DH EOS).

stiffness below approximately three nuclear densities, as well as its high-density behavior. As seen for example in Fig. 5, for our fixed magnetic dissipation evolution model, a stiff BM EOS with a high-density hyperon softening predicts a fractional increase of $n_{\mathrm{c}}$ by about $120 \%$ at $M_{\mathrm{i}}=1.4 M_{\odot}$, while $\mu_{\mathrm{c}}$ increases by $\sim 500 \mathrm{MeV}$. The overall picture obtained for magnetized stars, with $M_{\mathrm{i}}=1.2-1.6 M_{\odot}$, is therefore essentially different from the $B=0$ one.

\section{Conclusions}

We have studied the influence of two thin-disk accretion models - with and without the involvement of magnetic field - on the evolution of the central density and chemical potential in accreting NSs. For $B=0$ accretion from the marginally-stable circular orbit leads to a negligible central compression (a few per cent) for astrophysically-relevant initial configurations (slowlyrotating stars with $M_{\mathrm{i}}=1.2-1.6 M_{\odot}$ ) and maximally-efficient angular momentum transfer $\left(x_{1}=1\right.$ in Eq. (1), a value currently favored by numerical simulations). Consequently, this mechanism does not seem viable to trigger a dense-matter phase transition. Substantial central compression could be obtained for $x_{1}$ smaller than unity, or in a different evolutionary process (e.g., spin-down). Including the magnetic torque changes the outcome profoundly, but for a typical initial magnetic field, $B_{\mathrm{i}} \simeq 10^{12} \mathrm{G}$ and accretion rate $\dot{M}_{\mathrm{b}}=10^{-9} M_{\odot}$ one is able to produce massive, weakly-magnetized $\left(B \simeq 10^{8} \mathrm{G}\right)$ millisecond pulsars with a sizable (even as large as $100 \%$ ) central compression, thus probing much higher densities, which in turn may trigger a phase transition. Even such a low final value of $B$ still influences the accretion process and should be accounted for; only lowering it even more, to $\simeq 10^{7} \mathrm{G}$, essentially allows for the recovery of the $B=0$ results.

Note also a quite impressive dependence of the NS spin-up evolution on the very presence of the magnetic-field. The magnetic torque substantially decreases the spin-up efficiency, as the comparison with the $x_{1}=1$ and $x_{1}=0.5$ results show - in other words, spinning-up with magnetic field requires more accreted mass to reach a desired spin frequency. A considerable subset

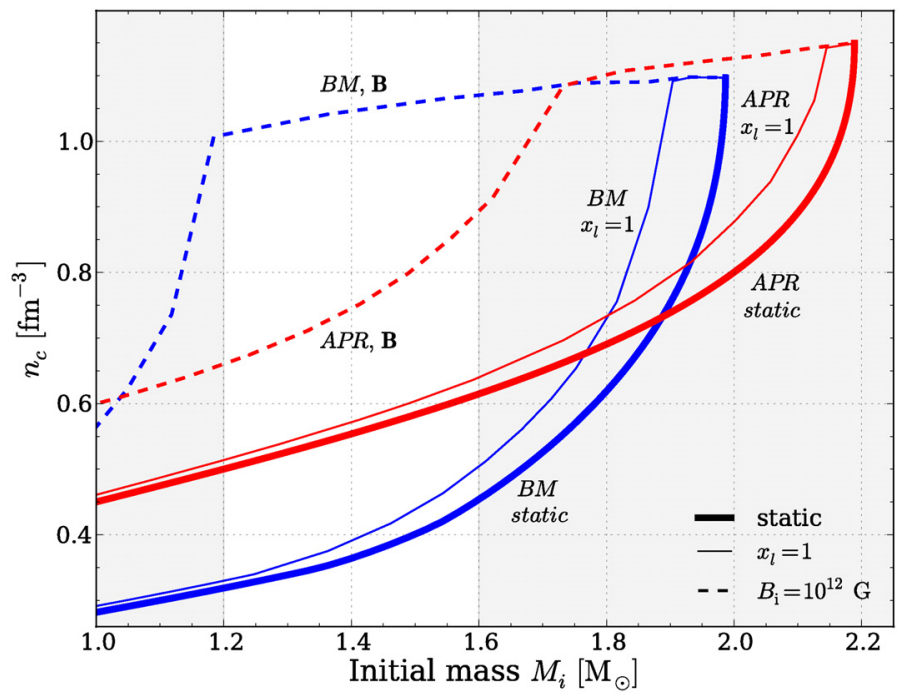

Fig. 5. Increase of the central baryon number density $n_{\mathrm{c}}$ as a function of the initial gravitational mass $M_{\mathrm{i}}$ for the APR and BM EOSs. Notations as in Fig. 3.

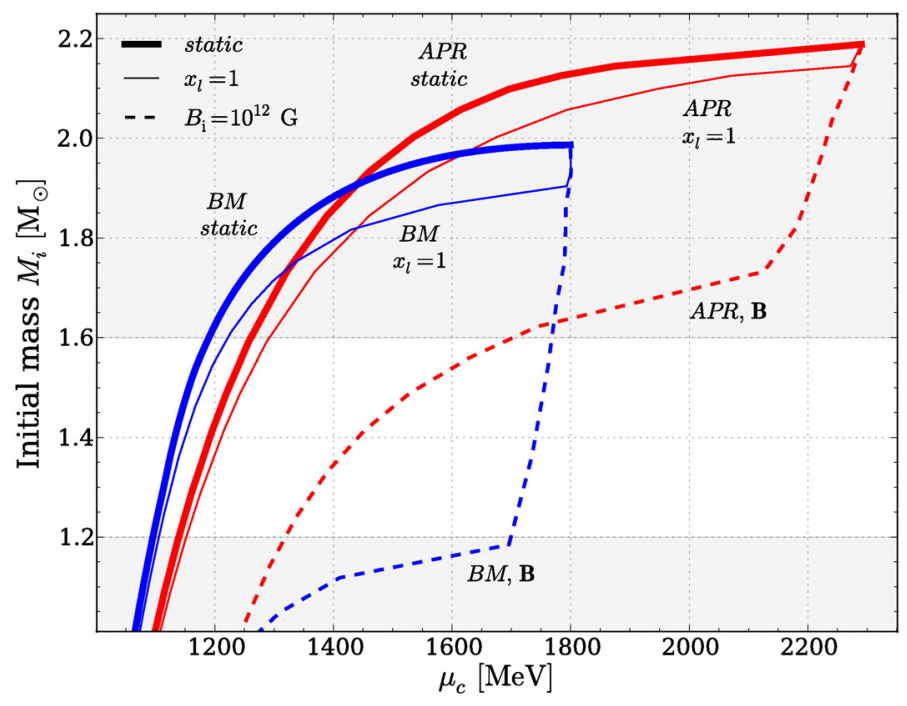

Fig. 6. initial gravitational mass $M_{\mathrm{i}}$ as a function of intial and maximal central chemical potentials, $\mu_{\mathrm{c}}$ for the APR and BM EOSs. Notations as in Fig. 4.

of "magnetic-torque" tracks ends at the axisymmetric instability limit (i.e., nearly horizontal lines in Figs. 3 and 5), while the vast majority of the $x_{1}=1$ tracks can reach the mass-shedding limit. This result correlates with the EOS stiffness and is most pronounced for the BM EOS, softened by the existence of hyperons; potentially, it may help in the understanding of a puzzling non-detection of submillisecond pulsars, as well as assist in the studies of formation of stellar-mass BHs and their mass function.

We have restricted ourselves to the EOSs of hadronic matter. A phase transition to quark matter softens the high-density EOS, so that reaching $M_{\max }>1.97 M_{\odot}$ requires some tuning of both hadronic and the quark-matter model. An approach based on an effective model of the QCD of quark matter used recently by Bonanno \& Sedrakian (2011) indicates that the vector repulsion in quark matter should be sufficiently strong to reach $M_{\max }>1.97 M_{\odot}$ (we recall that vector-meson repulsion in hadronic matter is also crucial for our BM EOS model). In any case, the maximum mass of NSs with quark cores (so-called hybrid stars) turns out to be very close to that reached at the 


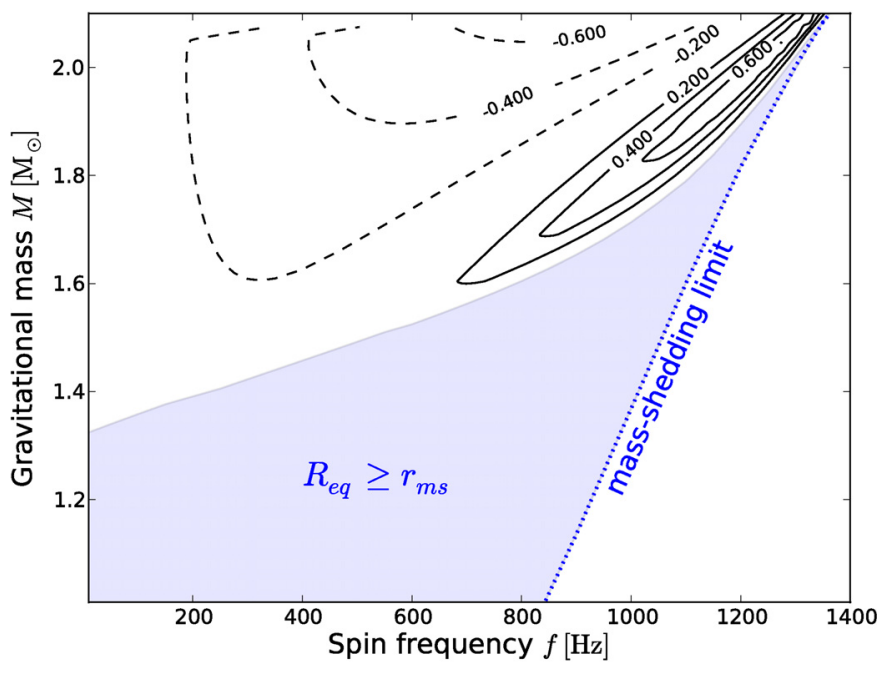

Fig. A.1. Difference (in $\mathrm{km}$ ) between the real value of $r_{\mathrm{ms}}$ and the value $r_{\mathrm{ms}}^{\text {(appr.) }}$ (to be compared with average radii of the problem: $R_{\mathrm{eq}}, r_{\mathrm{ms}} \sim$ $10 \mathrm{~km}$ ) obtained by means of an approximation of Bejger et al. (2010, 2011), plotted as iso-contours on the $M-f$ plane (DH EOS).

central density equal to the deconfinement density (Bonanno \& Sedrakian 2011).

Our results were obtained using three specific EOSs of dense matter, an effective magnetic torque model in the pulsaraccretion disk system, and a simplistic description of accretioninduced magnetic-field dissipation. We believe, however, that in a qualitative sense these results posses a general validity.

Acknowledgements. This work was partially supported by the Polish MNiSW research grant no. N N203 512838, LEA Astrophysics Poland-France (AstroPF) and ESF Research Networking CompStar programmes. M.B. acknowledges the Marie Curie Fellowship within the 7th European Community Framework Programme (ERG-2007-224793).

\section{Appendix A: Calculation of the inner-boundary radius of the accretion disk}

To simplify the calculation of the specific orbital angular momentum $l$ at every step of spin-up evolution, we used the Bejger et al. (2010) result: the Keplerian orbital frequency of a particle in the thin disk at a radius $r_{0}$ is well-approximated by the "Schwarzschildian/Newtonian" formula $\sqrt{G M / r_{0}}$. This ansatz yields a surprisingly accurate determination of $l$ for $r_{0} \simeq r_{\mathrm{ms}}$ for a broad range of stellar masses and spin frequencies up to the mass-shedding limit. Here we show that this approximate approach allows for a reasonably accurate determination of the $r_{\mathrm{ms}}$ as well. It corresponds to the $\mathrm{d} l / \mathrm{d} r=0$ condition; the inner-edge of an accretion disk $r_{0}$ is a solution of

$\frac{1}{2} f_{\mathrm{ms}}\left(r_{0}\right)=\left(\frac{r_{\mathrm{m}}}{r_{0}}\right)^{7 / 2}\left(\sqrt{\frac{r_{\mathrm{cor}}^{3}}{r_{0}^{3}}}-1\right)=\xi^{7 / 2} \omega^{-10 / 3}(1-\omega)$, where $r_{\text {cor }}$ is the corotation radius, $r_{\mathrm{m}}=(G M)^{-1 / 7} \dot{M}^{-2 / 7} \mu^{4 / 7}$ is the magnetospheric radius and $\xi=r_{\mathrm{m}} / r_{\mathrm{cor}}$. Function $f_{\mathrm{ms}}$ introduced by Bejger et al. (2011; reducing to $f_{\mathrm{ms}} \equiv 1$ in the Eq. (17) of Kluźniak \& Rappaport 2007) ensures a proper behavior of the torque near $r_{\mathrm{ms}}$

$f_{\mathrm{ms}}=\frac{1-\alpha / \bar{r}^{3 / 2}}{\left(1-v^{2} / c^{2}\right)^{3 / 2} \sqrt{1-1 / \bar{r}}}\left(\frac{\bar{r}-2}{\bar{r}-1}-2 \frac{v^{2}}{c^{2}}+\frac{3 \alpha}{\bar{r}^{3 / 2}-\alpha}\right)$,

with $\alpha=J c /\left(\sqrt{2} G M^{2}\right), \bar{r}=r_{0} / r_{s}$ and $r_{s}=2 G M / c^{2}$ denoting the Schwarzschild radius. Marginally stable circular orbit radius $r_{\mathrm{ms}}$ is then a solution of equation $f_{\mathrm{ms}}\left(r_{\mathrm{ms}}\right)=0$. Figure A.1 shows, using the example of the DH EOS (other EOSs we tested yield similar results) the comparison of the $r_{\mathrm{ms}}$ calculated exactly from integrals of motion with the approximate value $r_{\mathrm{ms}}^{\text {(appr.) }}$, obtained by means of Eq. (A.2). For almost all configurations the difference is less than $0.6 \mathrm{~km}$ (for $r_{\mathrm{ms}}<R$ we adopt $r_{\mathrm{ms}}=R$ ), and the regions where $\left|r_{\mathrm{ms}}-r_{\mathrm{ms}}^{\text {(appr.) }}\right|>0.6 \mathrm{~km}$ are small and well-confined near high-mass and mass-shedding, submillisecond rotation.

\section{References}

Akmal, A., Pandharipande, V. R., \& Ravenhall, D. G. 1998, Phys. Rev. C, 58, 1804

Beckwith, K., Hawley, J. F., \& Krolik, J. H. 2008, MNRAS, 390, 21

Bednarek, I., \& Manka, R. 2009, J. Phys. G: Nucl. Part. Phys., 36, 095201

Bejger, M., Zdunik, J. L., \& Haensel, P. 2010, A\&A, 520, 16

Bejger, M., Fortin, M., Haensel, P., \& Zdunik, J. L. 2011, A\&A, in press [arXiv: 1106.2432]

Blaschke, D., Grigorian, H., \& Poghosyan, G. 2001, Physics of Neutron Star Interiors, 578, 285

Blaschke, D., Poghosyan, G., \& Grigorian, H. 2008, AIPC, 1068, 191

Bonanno, L., \& Sedrakian, A. 2011, A\&A, in press, Doi: 10.1051/0004-6361/201117832

Bonazzola, S., Gourgoulhon, E., Salgado, M., \& Marc, J. A. 1993, A\&A, 278, 42

Burderi, L., Possenti, A., Colpi, M., et al. 1999, ApJ, 519, 285

Cheng, K. S., \& Dai, Z. G. 1998, ApJ, 492, 281

Colpi, M., Possenti, A., Popov, S., \& Pizzolato, F. 2001, Physics of neutron star interiors, ed. D. Blaschke, A. Sedrakian, \& N. K. Glendenning (Springer, LNP 578), 440

Cook, G. B., Shapiro, S. L., \& Teukolsky, S. A., 1994, ApJ, 423, L117

Demorest, P. B., Demorest, P. B., Pennucci, T., et al. 2010, Nature, 467, 1081

Douchin, F., \& Haensel, P. 2001, A\& A, 380, 151

Ghosh P., \& Lamb F. K. 1979, ApJ, 234, 296

Ghosh, P., \& Lamb, F. K. 1991, Neutron Stars: Theory and Observations, ed. J. Ventura, \& D. Pines (Dordrecht: Kluwer) 363

Glendenning, N. K., \& Weber, F. 2001, ApJ, 559, L119, Physics of Neutron Star Interiors, 578, 305

van den Heuvel, E. P. J., \& Bitzaraki, O. 1995, A\&A, 297, L41

Kluźniak, W., \& Rappaport, S. 2007, ApJ, 671, 1990

Lavagetto, G. Burderi, L., D’Antona, F., et al. 2004, MNRAS, 348, 73

Osłowski, S., Bulik, T., Gondek-Rosińska, D., \& Belczyński, K., 2011, MNRAS, 413, 461

Possenti, A., Colpi, M., Geppert, U., et al. 1999, ApJS, 125, 463

Shafee, R., McKinney, J. C., Narayan, R. et al. 2008, ApJ, 687, L25

Shibazaki, N., Murakami, T., Shaham, J., \& Nomoto, K. 1989, Nature, 342, 656

Taam, R. E., \& van den Heuvel, E. P. J. 1986, ApJ, 305, 235

Xu, R., \& Liang, E. 2009, Science in China G: Phys. Astron., 52, 315

Zdunik, J. L., Haensel, P., \& Gourgoulhon, E. 2002, A\&A, 381, 933

Zdunik, J. L., Haensel, P., \& Bejger, M. 2005, A\&A, 441, 207 